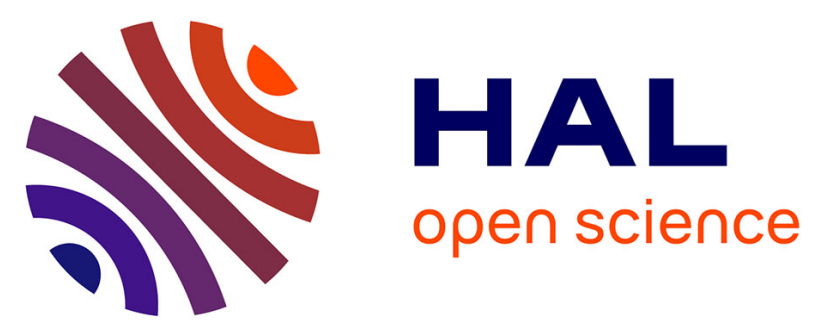

\title{
A Physiological Instability Displayed in Hippocampal Neurons Derived From Lithium-Nonresponsive Bipolar Disorder Patients
}

Shani Stern, Anindita Sarkar, Dekel Galor, Tchelet Stern, Arianna Mei, Yam Stern, Ana P.D. Mendes, Lynne Randolph-Moore, Guy A. Rouleau, Anne Bang, et al.

\section{To cite this version:}

Shani Stern, Anindita Sarkar, Dekel Galor, Tchelet Stern, Arianna Mei, et al.. A Physiological Instability Displayed in Hippocampal Neurons Derived From Lithium-Nonresponsive Bipolar Disorder Patients: Instability in bipolar disorder neurons. Biological Psychiatry, 2020, pp.S0006-3223(20)30055X. 10.1016/j.biopsych.2020.01.020 . inserm-02571200

\section{HAL Id: inserm-02571200 https://www.hal.inserm.fr/inserm-02571200}

Submitted on 12 May 2020

HAL is a multi-disciplinary open access archive for the deposit and dissemination of scientific research documents, whether they are published or not. The documents may come from teaching and research institutions in France or abroad, or from public or private research centers.
L'archive ouverte pluridisciplinaire HAL, est destinée au dépôt et à la diffusion de documents scientifiques de niveau recherche, publiés ou non, émanant des établissements d'enseignement et de recherche français ou étrangers, des laboratoires publics ou privés. 


\section{A physiological instability displayed in hippocampal neurons derived from lithium non-responsive bipolar disorder patients}

Shani Stern ${ }^{1,2}$, Anindita Sarkar¹, Dekel Galor ${ }^{1}$, Tchelet Stern' ${ }^{1}$, Arianna Mei ${ }^{1}$, Yam Stern', Ana P. D. Mendes ${ }^{1}$, Lynne Randolph-Moore ${ }^{1}$, Guy Rouleau ${ }^{2}$, Anne Bang ${ }^{3}$, Renata Santos ${ }^{1,5}$, Martin Alda ${ }^{4}$, Maria C. Marchetto ${ }^{1}$, Fred H. Gage ${ }^{1}$

1 Laboratory of Genetics, Gage, Salk Institute for Biological Studies, 10010 North Torrey Pines Road, La Jolla, CA 92037, USA

2 Sagol Department of Neurobiology, Faculty of Natural Sciences, University of Haifa, Haifa, 3498838, Israel.

2 Montreal Neurological Institute, McGill University, Montreal

3 Conrad Prebys Center for Chemical Genomics, Sanford Burnham Prebys Medical Discovery Institute, 10901 North Torrey Pines Road, La Jolla, CA 92037, USA

4 Department of Psychiatry, Dalhousie University, 5909 Veterans' Memorial Lane, Halifax, NS, B3H 2E2, Canada

5 Laboratory of Dynamic of Neuronal Structure in Health and Disease, Institute of Psychiatry and Neuroscience of Paris (UMR_S1266 INSERM, University of Paris), 102 rue de la Santé, 75014 Paris, France

Corresponding authors:

Shani Stern, sstern@univ.haifa.ac.il, sstern@salk.edu

Fred H. Gage, gage@salk.edu

Short title: Instability in bipolar disorder neurons

Keywords: bipolar disorder, CA3 pyramidal, dentate gyrus, numerical simulation, Physiological instability, computational model 


\begin{abstract}
Background:

We recently reported a hyperexcitability phenotype displayed in dentate gyrus (DG) granule neurons derived from bipolar disorder (BD) patients $(1,2)$ and also a hyperexcitability that appeared only in CA3 pyramidal hippocampal neurons that were derived from BD patients who responded to lithium treatment (LR) but not from BD patients who were lithium non-responders (NR) (3).
\end{abstract}

Methods:

Here we used our measurements of currents in neurons derived from 4 control individuals, 3 LR BD patients, and 3 NR BD patients. We have changed the conductances of simulated DG and CA3 hippocampal neurons according our measurements to derive a numerical simulation for BD neurons.

Results:

The computationally simulated BD DG neurons had a hyperexcitability phenotype similar to the experimental results. The simulated BD CA3 neurons were only hyperexcitable when derived from LR BD patients. Interestingly, our computational model captured a physiological instability intrinsic to hippocampal neurons that were derived from NR BD patients that we also observed when reexamining our experimental results. This instability is caused by a drastic reduction in the sodium current, accompanied by an increase in the amplitude of the fast potassium currents. These baseline alterations cause NR BD hippocampal neurons to drastically shift their excitability with small changes to their sodium currents, alternating between hyperexcitable and hypoexcitable states.

Conclusions:

Our computational model of BD hippocampal neurons that was based on our measurements reproduced the experimental phenotypes of hyperexcitability and physiological instability. We hypothesize that the physiological instability phenotype strongly contributes to affective lability in BD patients.

\title{
Introduction
}

Bipolar disorder (BD) is a devastating neuropsychiatric disease affecting 1-3\% of the general population, causing a major economic burden and drastically reducing the quality of life of patients. Induced pluripotent stem cell (iPSC) technology has allowed us to study BD in neurons derived from human patients (3-5).This approach was an exciting advance because it allowed viable cell studies in a disease for which animal models did not fully reconstruct the complicated genetics. Neither did these models fully recapitulate the symptoms of human patients $(6,7)$. Our first two studies in this line of research described in detail the phenotype that DG neurons derived from BD patients exhibited: hyperexcitability in the form of sustained activity. Our most recent study (3) describes the phenotype of another type of hippocampal neuron, the CA3 pyramidal neuron, and further unravels a mechanism that is responsible for the measured phenotypes.

In a previous study (5), we showed that the physiology of DG neurons derived from patients with a good outcome from lithium treatment was very different from the physiology of DG neurons of NR patients. In our recent study (3), we also show that only CA3 pyramidal neurons derived from LR patients are significantly hyperexcitable compared to CA3 pyramidal neurons derived from healthy controls; the respective CA3 neurons derived from lithium NR patients are not significantly hyperexcitable. Moving from the dish to human patients, it is interesting to note that the behavioral characteristics of LR patients 
are also very different from those of NR patients $(8,9)$. There are quite a few studies showing that patients who suffer from mixed episodes (depression and mania symptoms occurring at the same time) are less likely to respond to lithium treatment (10-14), and so mixed episodes mainly occur in lithium NR patients.

In this study we used our experimental data presented in $(3,5)$ to build a numerical simulation of the two types of studied hippocampal neurons: DG and CA3 pyramidal neurons. By increasing the conductance of a few types of the fast potassium currents and the sodium current, we were able to simulate BD LR and BD NR neurons for both DG and CA3 pyramidal neurons with the experimental phenotypes. Our computational models showed further evidence that BD hyperexcitability was linked with increased amplitude of potassium currents Moreover, we showed that NR hippocampal neurons displayed a physiological instability that resulted in a network of neurons in multi-excitatory states.

\section{Methods}

\section{Patients}

The cohort in this study consisted of the same patients used in the previous study (5). Supplementary table 1 summarizes their clinical data.

\section{Cell culture: DG neurons}

Using iPSC technology, DG granule neurons were cultured according to our published protocol (15) and measured at $\mathrm{t} 2=4.5$ weeks (Supplement).

\section{Cell culture: CA3 neurons}

CA3 pyramidal neurons were cultured according to our published protocol (16) and measured at $\mathrm{t} 2=4.5$ weeks.

\section{Whole cell patch clamp}

Neurons were infected with the ELAVL2::eGFP lentiviral vector at 15 days differentiation (as previously reported (16). Neurons on glass coverslips were transferred to a recording chamber in standard recording medium containing (in $\mathrm{mM}$ ) $10 \mathrm{HEPES}, 4 \mathrm{KCl}, 2 \mathrm{CaCl} 2,1 \mathrm{MgCl} 2,139 \mathrm{NaCl}$, and $10 \mathrm{D}$-glucose (310 mOsm, pH 7.4). Whole-cell patch-clamp recordings were performed from ELAVL2::eGFP-highlighted CA3 pyramidal neurons [the neurons patched were typically the larger cells in all the groups, which is customary in the field (17), with bright ELAVL2::eGFP expression], typically at 4.5 weeks after start of differentiation. Patch electrodes were filled with internal solutions containing (in mM) $130 \mathrm{~K}$-gluconate, 6 $\mathrm{KCl}, 4 \mathrm{NaCl}, 10 \mathrm{Na}$-HEPES, 0.2 K-EGTA, 0.3 GTP, $2 \mathrm{Mg}$-ATP, 0.2 cAMP, 10 Dglucose, 0.15\% biocytin and $0.06 \%$ rhodamine. The $\mathrm{pH}$ and osmolarity of the internal solution were brought close to physiological conditions ( $\mathrm{pH} 7.3,290-300 \mathrm{mOsmol}$ ) (pipette tip resistance was typically 10-15M 2 ). Signals were amplified with aMulticlamp700B amplifier and recorded with Clampex 10.2 software (Axon Instruments). Data were acquired at a sampling rate of $20 \mathrm{kHz}$ and analyzed using Clampfit-10 and the software package Matlab (2014b, The MathWorks Inc., Natick, MA, 2000). All measurements were conducted at room temperature.

\section{Analysis of electrophysiological recordings}

See Supplement

\section{Simulation}


A neuron simulation environment (18) was used for the mathematical models of the cells. A published, fully reconstructed CA3 neuron (19) was used to simulate a CA3 pyramidal neuron whereas, for the BD LR and BD NR neurons, several conductances of the ioninc currentsthat were altered in BD neurons (3)were changed accordingly. For a DG neuron, a published (20) cell morphology was used. Another type of ion channel was added to the model to represent the fast kinetics potassium channel Kv3.1 (21) to both DG and CA3 simulations, due to the altered expression of these channels (3).

\section{$\underline{\text { Results }}$}

\section{A computational model for a BD DG granule neuron and a CA3 pyramidal neuron recapitulates experimental phenotypes}

We have shown the involvement of potassium currents in the hyperexcitability phenotype in BD (3). We next used the changes that we measured in the currents respectively as changes to channel conductances in a numerical model for $\mathrm{BD}$. For a DG neuron, we downloaded a published DG neuron with a morphology shown in Figure 1A (20). We modified the conductance of 4 potassium channels and a sodium channel (Fig. 1B). We increased the delayed rectifier conductance by $15 \%$ in the LR neuron and $30 \%$ in the NR neuron (similar to the changes in our electrophysiological recordings summarized in table 2 in the Supplementary). Similarly, we increased the conductance of the M current, which is also a sustained potassium current, by $15 \%$ for LR neurons and $30 \%$ for NR neurons. Since the Kcnc genes were upregulated along with evident changes to the amplitude and kinetics of the potassium currents (3), we added an already published Kencn 1 conductance. The qPCR results exhibited a large fold change of the Kcnc genes in LR neurons, yet the electrophysiological measurements showed slightly faster kinetics and approximately a $15 \%$ increase in the sustained potassium currents (which are composed of other currents too and do not solely reflect the changes in the Kcnc1/2 related currents). We therefore increased the Kcnc related conductance in the LR simulated neuron by $75 \%$, Finally, we increased the cell size by $20 \%$ for NR neurons, similar to what we had observed in NR neurons at the time point of the recordings. These changes are summarized in the table shown in Figure 1B. When injecting currents into these two BD models (LR and NR), we observed how the simulated BD neurons recapitulated the experimental phenotype of hyperexcitability in the form of sustained activity. Figure 1C-E shows evoked potentials in response to different current injections in these neurons. In the top right corner is the number of evoked potentials measured with current steps of $0.15 \frac{\mathrm{mA}}{\mathrm{cm}^{2}}$, starting at $0.45 \frac{\mathrm{mA}}{\mathrm{cm}^{2}}$. The DG BD LR and NR neurons produced more action potentials than the DG control neurons, recapitulating the experimental hyperexcitability phenotype.

We similarly modeled a CA3 pyramidal neuron using a fully reconstructed, published cell depicted in Figure 2A (19). We changed the conductance of 4 types of ion channels according to our electrophysiological measurements (summarized in Supplementary table 3) and qPCR results and the cell size according to capacitance and morphological measurements (3) (Fig. 2B summarizes the changes). Again, in the Kcnc1/2 conductances, we had two indications, one from qPCR results, indicating a large fold change (but mRNA did not translate immediately to conductance changes), and the second from the increase in the sustained potassium currents (about a 20\% increase, but these contained other types of currents). It was clear that the conductance was increased, and we increased it in the LR simulation by $100 \%$ (due to the large fold change in qPCR). We wanted to see whether the phenotype of hyperexcitability would be recapitulated even with a smaller conductance change, so we ran the model with a 50\% increase in the Kcnc1/2 conductance as well. As can be seen from Supplementary Fig. 4, the hyperexcitability phenotype persisted. Figure 2C-E displays the evoked potentials in the 3 neuron types - control, LR and $\mathrm{NR}$ - in response to current injections. The numbers on the right-hand side represent the total number of 
action potentials, with 26 current injections starting at $0.45 \frac{\mathrm{mA}}{\mathrm{cm}^{2}}$ and with a current step of $0.15 \frac{\mathrm{mA}}{\mathrm{cm}^{2}}$. The computationally modeled CA3 LR neuron was hyperexcitable compared to the control neuron, but the NR neuron was not. The modeled CA3 BD neurons therefore also recapitulated the experimental phenotype.

\section{NR hippocampal neurons display a physiological instability both in the experiment and in a numerical simulation}

NR DG neurons in experiment and in simulation were, on average, hyperexcitable. However, these neurons also exhibited a form of change in their physiology that we termed a "physiological instability." We usually observed within the same culutre, neurons that were hyperexcitable, neurons that were hypoexcitable, and neurons with neurotypical excitability. To assess this phenomenon quantitatively, within each experiment, we defined a state score, describing the excitability of the neuron. The state score was ' 1 ' (hyperexcitable) to those cells that produced more than the average plus a half of a standard deviation of the number of action potentials that were evoked by the control neurons on average. The state score was -1 ('hypoexcitable') to those neurons that produced less than the average minus a half of the standard deviation action potentials than the control neurons. Anything in between received a state score of 0 and was considered 'neurotypical.' For each experiment, we calculated a diversity measure by the standard deviation of these state scores, as depicted in Figure 3A. Each dot in this plot represents a diversity in the neuronal excitability within a single experiment, calculated by the standard deviation of the state scores of each of the patched neurons (5-10 neurons within one experiment). The results indicated that there was a larger diversity in the excitability of NR neurons compared to control neurons.. Similarly (Fig. 3B), there was a larger diversity in the excitability of CA3 NR neurons compared to CA3 control neurons within an experiment.

Trying to understand why NR neurons had a larger diversity in the excitability, we noticed a strong influence of the sodium currents. First, when we measured the correlation between excitability and sodium currents (3), this correlation was very significant, and the slope of the graph was very steep for NR neurons but much less significant for the other groups ((3)Fig. 3C-E). Second, we calculated the sodium currents (at $0 \mathrm{mV}$ ) for the hypoexcitable neurons and compared them to the sodium currents (at $0 \mathrm{mV}$ ) of the rest of the recorded cells. The difference between these 2 groups was drastic ( $\mathrm{p}=3 \mathrm{e}-5$ for DG neurons Fig. $3 \mathrm{Cc}$, and $\mathrm{p}=4 \mathrm{e}-5$ for $\mathrm{CA} 3$ neurons, Fig. 3D), indicating that hypoexcitable neurons observed in the NR group exhibited low sodium currents.

Example recordings illustrating the larger diversity of excitability in NR neurons are provided in Fig. 3EH. Fig. 3E presents one experiment with control neurons. The number of action potentials did not vary greatly between the different recorded neurons in the control experiment. In the left corner of each evoked potential plot the inset shows the sodium currents at $-20 \mathrm{mV}$ and $-10 \mathrm{mV}$. Fig. 3F presents one experiment with NR neurons. A big diversity in excitability is evident with a large proportion of hypoexcitable or hyperexcitable neurons, and we termed this behavior a "mixed physiological state".. The sodium currents in the inset show the strong influence of these currentson the excitability of NR neurons; the hypoexcitable cells usually had very low sodium currents. This mixed physiological state represented most of our NR experiments ( $71 \%$ of NR experiments compared to $33 \%$ of control experiments). However, the network of NR neurons also sometimes organized in a general "hypoexcitable state," where almost all the measured neurons within the experiment were hypoexcitable and had almost no evoked action potentials (28\% in NR experiments compared to $0 \%$ in control experiments) (example experiment Fig. $3 \mathrm{G}$ ). The sodium currentsin the inset strongly suggest that this global hypoexcitability stemmed from very low sodium currents globally in the culture. Interestingly, we were also able to find experiments where the NR network was in a global "hyperexcitability state" (17\% in NR experiments compared to 5\% in control experiments), where most 
neurons in the experiment were hyperexcitable (Fig. 3H). Even in this hyperexcitable state, the few NR neurons that were hypoexcitable had very low sodium currents.

To summarize, NR DG neurons had lower sodium current amplitudes on average (3), but at this low sodium level they were hyperexcitable on average. However, due to the natural diversity between the cells, there were always neurons with lower than average sodium currents and; these cells were extremely hypoexcitable. We termed this a physiological instability because shifts in the sodium currents resulted in a large change in the excitability of the cells. We further hypothesized that this phenomenon likely contributes to depression (the global hypoexcitability state), mania (the global hyperexcitability state), and mixed episodes (the mixed physiological state).

Similar analysis of CA3 neurons also showed a clear physiological instability of the NR CA3 neurons. The standard deviations of the hyperexcitability scores in each experiment are plotted in Figure 3B. The global hypoexcitability state occurred in $24 \%$ of the NR experiments and only in $12 \%$ of the control experiments. Global hyperexcitability occurred in $24 \%$ of the NR experiments and only in $6 \%$ of the control experiments. The mixed state occurred in $71 \%$ of NR experiments and $53 \%$ of the control experiments

We then turned to the numerical simulation. Since we observed an experimental physiological instability in NR neurons (both DG and CA3) that was dependent on the sodium currents, we were interested to see how the excitability of the numerically simulated neurons (NR compared to control) changed when adding a perturbation to the sodium conductance and deviating it around its mean values, similar to the natural variation in the conductance that we see in the experiment.. We deviated the sodium conductance of the numerically simulated control and NR neurons by constant steps around the mean conductance. As can be observed from Figure 3I, the BD NR neuron changed its excitability with a larger derivative with respect to changes in sodium conductance (steeper slope); therefore, it had a higher tendency to become hypoexcitable or hyperexcitable upon deviations in sodium currents (using an ANOVA, we calculated a significant change in the slope of the NR vs. control curve, $\mathrm{p}=0.0034)$. Thus, in its basal state of lower amplitudes of sodium currents and higher amplitudes of potassium currents, the NR DG neuron was physiologically unstable also in the numerical simulation; small changes in its sodium currents were translated into large changes in its excitability. We next simulated the changes in the sodium conductance of the NR and control CA3 neurons and observed a similar physiological instability in the NR neurons compared to control. The slope of the excitability as a function of the sodium conductance was steeper for the NR neurons ( $\mathrm{p}=0.001$ using an ANOVA), showing also a physiological instability in NR CA3 numerically simulated neurons. The changes in the cells' excitability as a function of other ion channels perturbations are presented in Supplementary Fig. 1,2.

\section{Potassium channel blockers reduce the percentage of non-typically spiking NR neurons.}

We have shown (3)that application of $1 \mathrm{mM}$ TEA or $200 \mathrm{nM}$ DTX or $0.3 \mathrm{mM} 4$-AP reduces the hyperexcitability of LR CA3 neurons. In NR CA3 neurons, 4-AP significantly reduced the excitability and DTX and TEA reduced the excitability, but not significantly. The phenotype of NR neurons was more complicated and involved multi-excitatory states of the neurons. We tested the effects of 4-AP, DTX and TEA on the distribution of excitability in the NR cultures. We defined 3 states in each recorded neuron: very hypoexcitable (not being able to produce action potentials at all), hyperexcitable (more than the mean number of action potentials in the control neurons plus a half of standard deviation of the number of action potentials in the control), or neurotypical (anything within that range). The distribution over these states in control and NR CA3 neurons is presented in Figure 4A. Similarly, this distribution is presented for control vs NR after application of $0.3 \mathrm{mM} 4-\mathrm{AP}$ in Figure 4B. The excitability distributions with application of 1 mM TEA and $200 \mathrm{nM}$ DTX are presented in Figure 4C and D, respectively. The distributions in Fig. 4 
indicate that application of potassium channel blockers changed the excitability states of NR neurons to be closer to that of the controls. When comparing the percentage of non-typically spiking neurons (plotted in the top right of each of the distributions), there is a significant increase in non-typical neurons in NR neurons compared to control neurons (Fig. 4A, $\mathrm{p}=0.0097$ ), but no change in NR neurons treated with potassium channel blockers compared to control neurons (4B-D).

\section{Discussion}

The introduction of induced pluripotent stem cell (iPSC) technology allowed us to recognize a clear hyperexcitability physiological phenotype of DG neurons derived from BD patients $(1,4)$ and the hyperexcitability of CA3 hippocampal pyramidal neurons derived from LR BD patients (3). Other iPSC studies have shown that expression of transcripts for membrane-bound receptors and ion channels increased in BD neurons, and BD neurons treated with lithium exhibited a smaller amplitude of calcium transients (22). Not many other functional studies have been performed, but GWAS studies also suggest ion channelopathies may be involved in BD (23). Here we focus our attention on an intrinsic physiological instability in NR neurons, shifting their excitability drastically with small changes in their sodium currents, which results in a network with multi-excitatory states and suggests the existence of multi-excitatory states in the patients' brains that likely contribute to the patients' emotional dysregulation.

In order to design computational models for the two types of BD hippocampal neurons that we have extensively characterized using whole cell patch clamp (3-5), we altered conductances of potassium and sodium channels in the simulated BD neurons with respect to the control similar to the changes in the related currents amplitudes in our experimental measurements. We also changed the size of the BD neurons to reflect the changes in morphology and capacitance that we measured in the BD neurons $(3,5)$. Interestingly, the physiological phenotype was recapitulated in our computational models; In current clamp mode, both LR and NR DG simulated neurons produced more total action potentials than the control DG neurons, whereas only the CA3 LR simulated neurons produced more total action potentials than the control CA3 neurons, like our experimental measurements (3). When comparing the simulated potassium currents in voltage clamp model, we see similar overall changes in the amplitudes of both the fast and slow components when compared to the example traces and also when comparing to the mean currents recorded over many neurons (3). The kinetics of the simulated currents differ from the recordings: the slow currents in the recorded neurons slowly decrease until stabilization, while in the simulation, the currents first decrease to a minimum at around $100 \mathrm{~ms}$. and then slowly increase until they stabilize. It is therefore important to note that although many of different ionic conductances are taken into account in the numerical simulation, it is not a complete replica of the live neuron and many types of ionic conductances were not implemented, similar to the original models that our simulations were based on $(19,20)$. For example, our simulation does not include $\mathrm{HCN}$ or inward rectifier channels. Therefore, the simulation does not fully replicate the complete neurophysiology of a live neuron, and we expect some differences. Despite this drawback, the Neuron simulation environment is extensively used to model different neurons (24-26), although imperfectly, concentrating usually on a specific set of ionic conductances of interest. Overall, our numerical models of BD neurons indicate that our observed findings on current amplitude, which we translated into changes in ion channel conductivity, are generally compatible with the observed alterations in overall excitability that we observed in the experiment and the simulation. Goldman et al. (27) have shown that similar patterns of firing can be established from different amplitudes and combinations of ionic currents. It is therefore important to keep in mind that these numerical models give additional support to our experimental conclusions (3), and the computational model together with the experimental results suggest that changes in the potassium currents are correlated and causative of the hyperexcitability of DG BD neurons, and of the hyperexcitability of CA3 LR neurons. 
While performing patch clamp of hundreds of neurons from the NR cohort, we recognized that the phenotype was more complicated than we initially reported; On average NR neurons were hyperexcitable, but many recorded neurons were hypoexcitable. . This phenomenon was not observed in the control and LR BD neurons, although they were grown in the same conditions. To quantify this large diversity in excitability states in NR neurons, we gave an excitatory score to each recorded neuron within the same experiment. The diversity of these scores was significantly higher in NR neurons compared to the controls. In a neuronal culture, there is a continuum range of values of the different currents (the law of large numbers dictates a continuous Normal distribution), but the already low average sodium current in NR hippocampal neurons resulted in those neurons on the lower part of this normal distribution of sodium currents being extremely hypoexcitable. However, due to the large amplitude of the fast potassium currents, the neurons with the average or higher-than-average sodium currents were usually hyperexcitable, resulting in a large number of hyperexcitable and hypoexcitable neurons within the same NR culture. Potassium channel blockers reduces the average excitability of NR hippocampal neurons and also importantly shifted many of the hypoexcitable neurons towards the neurotypical state, making the distribution of cell excitability closer to that of the controls.

We returned to the numerical simulation, to see if this physiological instability would be evident. We simulated a continuum of sodium channel conductances around the mean value that was originally used. Interestingly, deviating the sodium conductances resulted in large changes in neuronal excitability only in the NR neurons. NR neurons with low sodium conductances were hypoexcitable compared to control neurons with the same shift in their sodium conductance, whereas NR neurons with the higher sodium conductances were hyperexcitable compared with the same shift in sodium current in control. The intrinsic physiological instability that we observed in the experimental data was also therefore evident in the numerical simulation. NR neurons easily alternated between hypoexcitability and hyperexcitability states, with the same changes in sodium conductances that had little effect on the control neuron.

To understand the impact of this instability on the network behavior in the experiment, we explored the NR network organization was at different days of recordings (in mature cultures). In about $25 \%$ of the experiments, the NR network organized in a global hyperexcitability. In another $25 \%$, the network organized in a global hypoexcitability, and on most of the recording days, the network appeared to be in the multi-excitatory state, exhibiting neurons that were hyperexcitable, hypoexcitable and neurotypical all within the same dish. Witnessing these different states that occurred only in NR neurons, we hypothesized that these states contributed to behavioral symptoms such as mixed episodes (our mixed physiological state), depression (our global hypoexcitability state) and mania (our global hyperexcitability state). Linking this phenomenon to BD patients, it is useful to note that the presence of "mixed episodes" (manic and depressive symptoms that are present at the same time in a patient) is one of the clinical features that is more characteristic of patients who are non-responsive to lithium (12-14).

To summarize, this study complements our study of the mechanisms that underlie the hyperexcitability in BD hippocampal neurons (3). The numerical model provides further support to our conclusions that increased potassium currents participate in BD hippocampal neuronal hyperexcitability. Additionally, we report that DG and CA3 pyramidal hippocampal neurons derived from NR patients, exhibited an intrinsic physiological instability, shifting their excitability states easily. Their multi-excitatory state network occasionally shifts towards a global hyperexcitable or global hypoexcitable state, and usually exhibits irregular and extreme excitability states within the same dish.

Financial disclosures: The authors declare no biomedical financial interests or potential conflicts of interest. 


\section{Figure legends}

Figure 1. A NEURON computational model for DG control, BD LR and BD NR neurons recapitulates the electrophysiological phenotypes. The $\mathrm{BD}$ neurons were achieved by changing the ion channel conductance compared to the control neurons, similar to the changes that we observed in electrophysiology or qPCR. A. The morphology of the DG neuron that was simulated (20). B. A schematic showing how the different measured cellular changes affect the model. The table summarizes the changes in ion channel conductance and sizes of control, LR and NR DG neurons that were incorporated in the DG neuron simulation of the 3 groups. C-E. Evoked potentials in A. control, B. BD LR, and C. BD NR DG neurons. The number at the top right corner is the sum of the evoked potentials. BD DG neurons (LR and NR) display a hyperexcitability in the computational model similar to the experimental phenotype that we measured with patch clamp. F. Representative recorded currents in DG neurons from each of the groups (control, LR and $\mathrm{NR}$ ) in voltage clamp mode (clamping at $-60 \mathrm{mV} \rightarrow 20 \mathrm{mV} \rightarrow-60 \mathrm{mV}$ ) demonstrate an increase in outward potassium currents in BD neurons. G. Total somatic currents in the DG neurons' simulation (multiplying the current density by the mean soma surface area) in voltage clamp mode. The potentials used for the simulation were similar to those in the example recording (F), but after compensation of the liquid junction potential in the recordings (simulation clamping at $-70 \mathrm{mV} \rightarrow 20 \mathrm{mV} \rightarrow-70 \mathrm{mV}$ ). An increase in the amplitude of the outward currents is observed in the BD neurons, similar to the experimental results.

\section{Figure 2.}

A NEURON computational model for CA3 control, BD LR and BD NR neurons recapitulates the electrophysiological phenotypes. The BD neurons were achieved by changing the ion channel conductance compared to the control neurons, similar to the changes that we observed in electrophysiology or qPCR. A. The morphology of the CA3 neuron that was simulated (19). B. A schematic showing how the different measured cellular changes affect the model. The table summarizes the changes in ion channel conductance and sizes of control, LR and NR CA3 neurons that were implemented in the CA3 neuron simulation of the 3 groups. C-E. Evoked potentials in A. control, B. BD LR, and C. BD NR CA3 neurons. The number at the top right corner is the sum of the evoked potentials. BD LR CA3 neurons display a hyperexcitability in the computational model similar to the experimental phenotype that we measured with patch clamp. F. Representative recorded currents in CA3 neurons from each of the groups (control, LR and NR) in voltage clamp mode (clamping at $-60 \mathrm{mV} \rightarrow 20 \mathrm{mV} \rightarrow-60 \mathrm{mV}$ ) demonstrate an increase in outward potassium currents in the BD neurons. G. Total somatic currents in the CA3 pyramidal neurons' simulation (multiplying the current density by the mean soma surface area) in voltage clamp mode. The potentials were similar to those in the example recording $(\mathrm{F})$, but after compensation of the liquid junction potential in the recordings (simulated clamping at $-70 \mathrm{mV} \rightarrow 20 \mathrm{mV} \rightarrow-70 \mathrm{mV}$ ). An increase in the amplitude of the outward currents is observed in the BD neurons, similar to the experimental results.

Figure 3. A large diversity in excitability is observed in NR hippocampal neurons due to a physiological instability. Electrophysiological measurements $(\mathrm{A}-\mathrm{H})$ compared to the computational simulation (I-J). A. Measuring the diversity in excitability within each experiment (see Supplementary Methods) reveals that, in NR DG neurons, there is a significantly larger diversity in excitability between different neurons during an experiment as compared to control DG neurons. B. Measuring the diversity in excitability in each experiment (see Supplementary Methods) reveals that, in NR CA3 neurons, there is a significantly larger diversity in excitability between different neurons during an experiment as compared to control CA3 neurons. C. Dividing the NR DG neurons that we recorded in experiment into hypoexcitable neurons vs. 
all the rest, there is a very large and significant change in the sodium currents of the hypoexcitable neurons vs. all the rest. D. Dividing the NR CA3 neurons that we recorded into hypoexcitable neurons vs. all the rest, there is a very large and significant change in the sodium currents of the hypoexcitable neurons vs. all the rest. E-H. Examples of different experimental days recordings from a control (physiologically stable) DG culture and from a DG NR (physiologically unstable) culture. On each line, recordings during one experiment are plotted. The evoked potentials are presented in the main graph, and in the inset in the left corner, the normalized sodium currents are presented at $-20 \mathrm{mV}$ and $-10 \mathrm{mV}$. E. An example of a control culture in which neurons are not hyperexcitable or hypoexcitable. The total evoked potentials do not change much from neuron to neuron during the experiment. The mean sodium currents vary from around $15 \mathrm{pA} / \mathrm{pF}$ to $33 \mathrm{pA} / \mathrm{pF}$. F. Similarly, an example with recordings from several NR neurons during the same experiment is presented. Neurons tend to be hyperexcitable or hypoexcitable ("mixed physiological state"), where a main effector is the sodium current; the hypoexcitable neurons have sodium currents that are close to 0 . G. Although most of the NR networks tend to be in a mixed state of hyperexcitable and hypoexcitable cells, in approximately $15 \%$ of the experiments we found the entire culture was hypoexcitable, with neurons producing very little action potentials (and the sodium currents were very low throughout the experiment). Although most of the NR networks tend to be in a mixed state of hyperexcitable and hypoexcitable cells, in approximately $12 \%$ of the experiments we found the entire culture was hyperexcitable, with most neurons producing many action potentials (above the neurotypical- mean plus a half of a standard deviation). I. Applying changes to the sodium conductance in the numerical simulation of the DG neurons (as these vary throughout the different neurons in the experimental settings) causes mild changes in excitability of the control DG neuron (blue) vs. drastic changes in excitability in the BD NR DG neuron (green). J. Applying changes to the sodium conductance in the numerical simulation of the CA3 neurons (as these vary throughout the different neurons in the experimental settings) causes mild changes in excitability of the control CA3 neuron (blue) vs. drastic changes in excitability in the BD NR CA3 neuron (green). We conclude that low sodium currents are the main driver of the large number of hypoexcitable neurons that we observe in NR cultures. Note that NR neurons have a significantly reduced sodium current ((3)Fig. 2). In the experiment, like in the simulation, NR neurons with the average (decreased) sodium currents compared to control neurons are on average hyperexcitable, but those neurons with sodium currents that are below the average (already very reduced) sodium current become hypoexcitable. We termed this phenomenon a "physiological instability," since the NR neurons are easily shifted between a hyperexcitable and a hypoexcitable state. Asterisks represent statistical significance by the following code: $* \mathrm{p}$ value $<0.05$, $* * * \mathrm{p}$ value $<0.001, * * * * \mathrm{p}$ value $<0.0001$. Error bars represent standard error.

Figure 4. Potassium channel blockers reduce the percentage of non-typically spiking NR neurons. A. A comparison of the distribution of the excitability of control and NR neurons into 3 different states hypoexcitable, neurotypical and hyperexcitable. Inset of the top right shows that there is a significant increase in the number of non-typically spiking neurons in the NR networks compared to the control networks. B. A comparison of the distribution of the excitability of control and NR neurons with 4-AP into 3 different states - hypoexcitable, neurotypical and hyperexcitable. Inset on the right shows that, in NR networks with 4-AP applied, the amount of non-typically spiking neurons is not significantly different than in the control networks. C. A comparison of the distribution of the excitability of control and NR neurons with TEA into 3 different states - hypoexcitable, neurotypical and hyperexcitable. Inset on the right shows that, in NR networks with TEA applied, the amount of non-typically spiking neurons is not significantly different than in the control networks. D. A comparison of the distribution of the excitability of control and NR neurons with DTX into 3 different states - hypoexcitable, neurotypical and hyperexcitable. Inset on the right shows that, in NR networks with DTX applied, the amount of non-typically spiking neurons is not significantly different than in the control networks Overall these results indicate that potassium channel 
blockers shift the distribution of the excitability of NR neurons to be closer to that of the controls. Asterisks represent statistical significance by the following code: ${ }^{*} \mathrm{p}$ value $<0.05$.

\section{Figures}

Figure 1

A

B
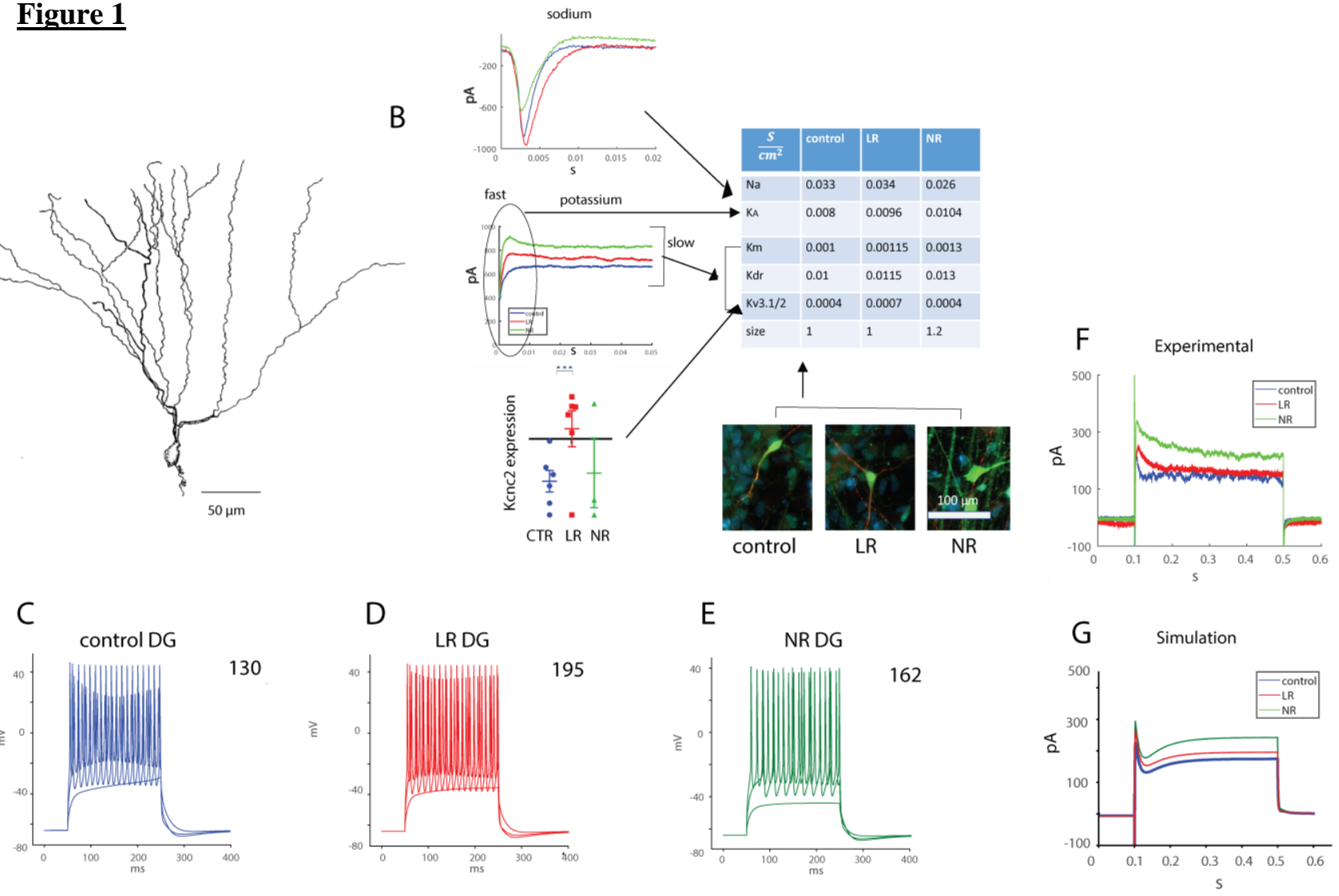

Figure 2 
A

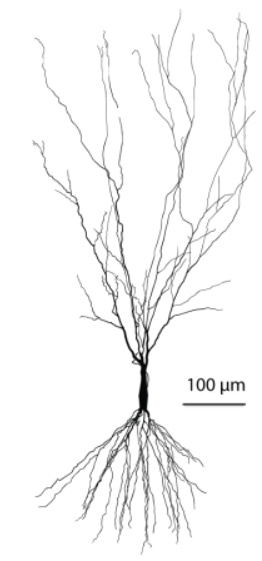

C control CA3

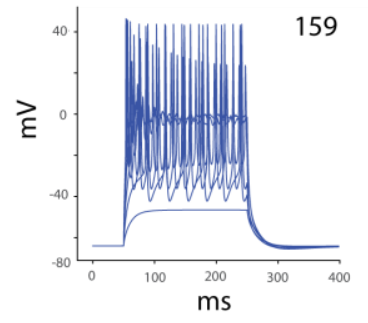

B
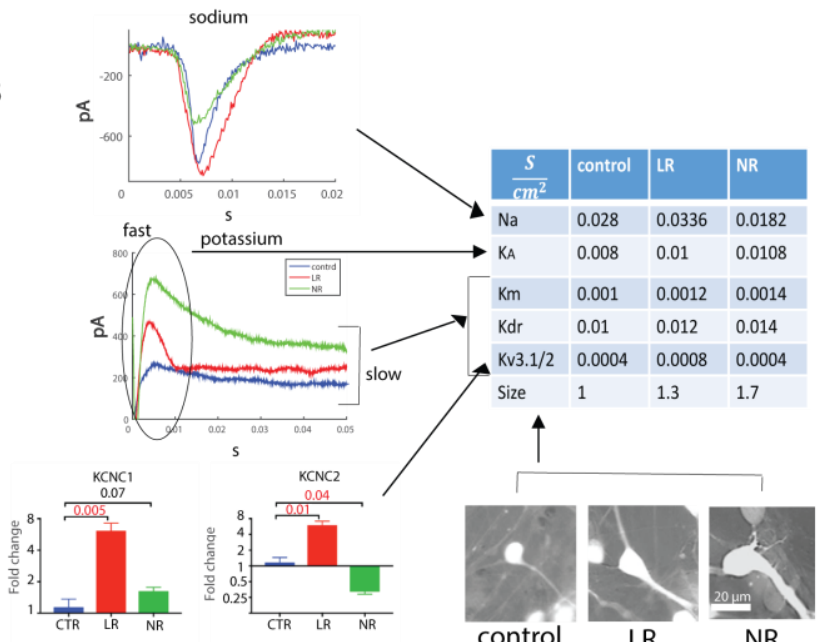

D LRCA3

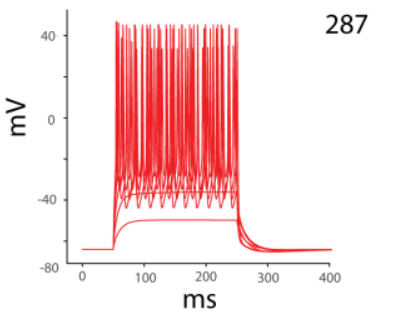

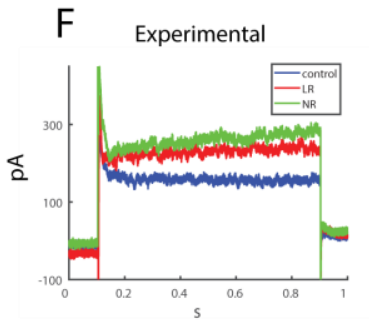

E
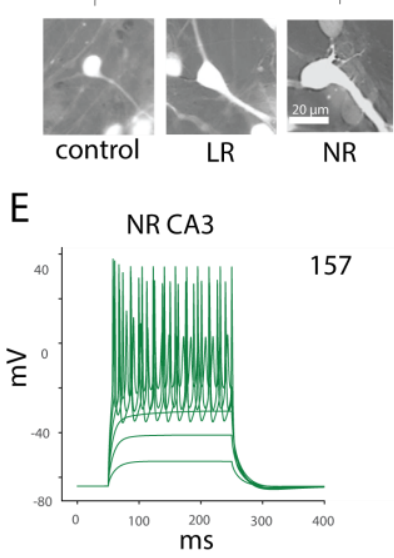

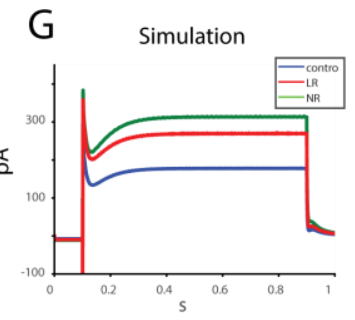

Figure 3 

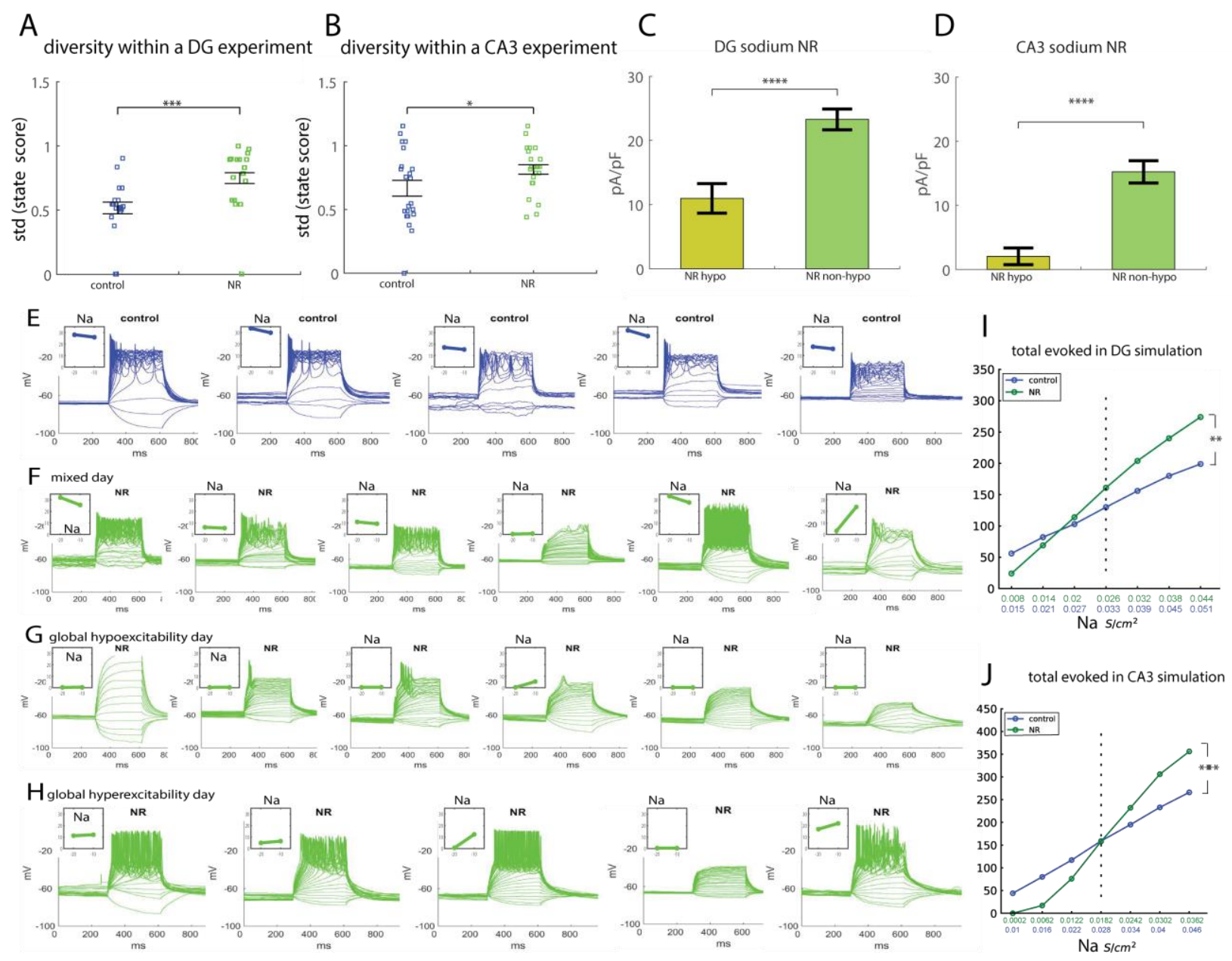

Figure 4 

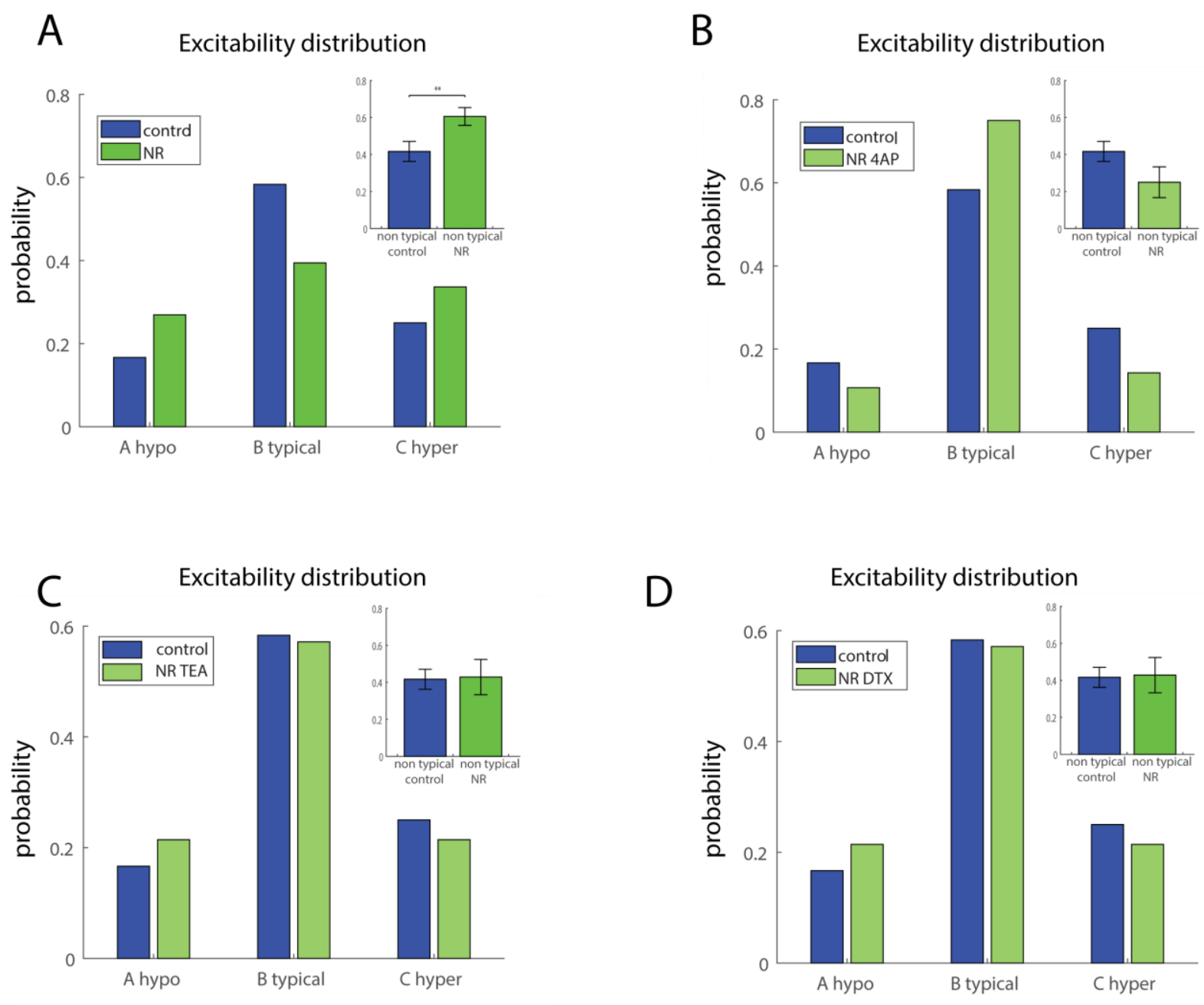

\section{Acknowledgments}

This work was supported by the National Cancer Institute Grant No. P30 CA014195, the National Institutes of Health, R01 AG05651. The authors would like to thank the National Cooperative Reprogrammed Cell Research Groups (NCRCRG), grant number U19 MH106434. The Gage laboratory is supported in party by the Leona M. and Harry B. Helmsley Charitable Trust \#2017-PG-MED001, the JPB Foundation, Annette C. Merle-Smith, and the Robert and Mary Jane Engman Foundation. The lymphoblast samples and the clinical data were obtained with support from Canadian Institutes of Health Research Grant No. \#64410 (to MA and GR).

\section{$\underline{\text { References }}$}

1. Stern S, Santos R, Marchetto MC, Mendes AP, Rouleau GA, Biesmans S, Wang QW, Yao J, Charnay P, Bang AG, Alda M, Gage FH (2017): Neurons derived from patients with bipolar disorder divide into 
intrinsically different sub-populations of neurons, predicting the patients' responsiveness to lithium. Molecular psychiatry.

2. Mertens J, Wang QW, Kim Y, Yu DX, Pham S, Yang B, Zheng Y, Diffenderfer KE, Zhang J, Soltani S, Eames T, Schafer ST, Boyer L, Marchetto MC, Nurnberger JI, Calabrese JR, Odegaard KJ, McCarthy MJ, Zandi PP, Alda M, Nievergelt CM, Pharmacogenomics of Bipolar Disorder S, Mi S, Brennand KJ, Kelsoe JR, Gage FH, Yao J (2015): Differential responses to lithium in hyperexcitable neurons from patients with bipolar disorder. Nature. 527:95-99.

3. Stern S, Sarkar A, Stern T, Mei A, Mendes APD, Stern Y, Goldberg G, Galor D, Nguyen T, RandolphMoore L, Kim Y, Rouleau G, Bang A, Alda M, Santos R, Marchetto MC, Gage FH (2019): Mechanisms Underlying the Hyperexcitability of CA3 and Dentate Gyrus Hippocampal Neurons Derived From Patients With Bipolar Disorder. Biol Psychiatry.

4. Mertens J, Paquola ACM, Ku M, Hatch E, Bohnke L, Ladjevardi S, McGrath S, Campbell B, Lee H, Herdy JR, Goncalves JT, Toda T, Kim Y, Winkler J, Yao J, Hetzer MW, Gage FH (2015): Directly Reprogrammed Human Neurons Retain Aging-Associated Transcriptomic Signatures and Reveal AgeRelated Nucleocytoplasmic Defects. Cell stem cell. 17:705-718.

5. Stern S, Santos R, Marchetto MC, Mendes APD, Rouleau GA, Biesmans S, Wang QW, Yao J, Charnay P, Bang AG, Alda M, Gage FH (2018): Neurons derived from patients with bipolar disorder divide into intrinsically different sub-populations of neurons, predicting the patients' responsiveness to lithium. Mol Psychiatry. 23:1453-1465.

6. $\quad$ Beyer DKE, Freund N (2017): Animal models for bipolar disorder: from bedside to the cage. Int J Bipolar Disord. 5:35.

7. Nestler EJ, Hyman SE (2010): Animal models of neuropsychiatric disorders. Nature neuroscience. 13:1161-1169.

8. Stern S, Linker S, Vadodaria KC, Marchetto MC, Gage FH (2018): Prediction of response to drug therapy in psychiatric disorders. Open biology. 8.

9. Tighe SK, Mahon PB, Potash JB (2011): Predictors of lithium response in bipolar disorder. Ther Adv Chronic Dis. 2:209-226.

10. Fountoulakis KN, Kontis D, Gonda X, Siamouli M, Yatham LN (2012): Treatment of mixed bipolar states. Int J Neuropsychopharmacol. 15:1015-1026.

11. Sportiche S, Geoffroy PA, Brichant-Petitjean C, Gard S, Khan JP, Azorin JM, Henry C, Leboyer M, Etain B, Scott J, Bellivier F (2017): Clinical factors associated with lithium response in bipolar disorders. Aust N Z J Psychiatry. 51:524-530.

12. Etain B, Lajnef M, Brichant-Petitjean C, Geoffroy PA, Henry C, Gard S, Kahn JP, Leboyer M, Young AH, Bellivier $F(2017)$ : Childhood trauma and mixed episodes are associated with poor response to lithium in bipolar disorders. Acta psychiatrica Scandinavica. 135:319-327.

13. Kruger S, Trevor Young L, Braunig P (2005): Pharmacotherapy of bipolar mixed states. Bipolar Disord. 7:205-215.

14. Backlund L, Ehnvall A, Hetta J, Isacsson G, Agren H (2009): Identifying predictors for good lithium response - a retrospective analysis of 100 patients with bipolar disorder using a life-charting method. Eur Psychiatry. 24:171-177.

15. Yu DX, Di Giorgio FP, Yao J, Marchetto MC, Brennand K, Wright R, Mei A, McHenry L, Lisuk D, Grasmick JM, Silberman P, Silberman G, Jappelli R, Gage FH (2014): Modeling hippocampal neurogenesis using human pluripotent stem cells. Stem Cell Reports. 2:295-310.

16. Sarkar A, Mei A, Paquola ACM, Stern S, Bardy C, Klug JR, Kim S, Neshat N, Kim HJ, Ku M, Shokhirev MN, Adamowicz DH, Marchetto MC, Jappelli R, Erwin JA, Padmanabhan K, Shtrahman M, Jin X, Gage FH (2018): Efficient Generation of CA3 Neurons from Human Pluripotent Stem Cells Enables Modeling of Hippocampal Connectivity In Vitro. Cell Stem Cell. 22:684-697 e689. 
17. Stern S, Segal M, Moses E (2015): Involvement of Potassium and Cation Channels in Hippocampal Abnormalities of Embryonic Ts65Dn and Tc1 Trisomic Mice. EBioMedicine. 2:1048-1062.

18. Hines ML, Carnevale NT (1997): The NEURON simulation environment. Neural computation. 9:1179-1209.

19. Migliore M, Cook EP, Jaffe DB, Turner DA, Johnston D (1995): Computer simulations of morphologically reconstructed CA3 hippocampal neurons. Journal of neurophysiology. 73:1157-1168.

20. Aradi I, Holmes WR (1999): Role of multiple calcium and calcium-dependent conductances in regulation of hippocampal dentate granule cell excitability. J Comput Neurosci. 6:215-235.

21. Wang LY, Gan L, Forsythe ID, Kaczmarek LK (1998): Contribution of the Kv3.1 potassium channel to high-frequency firing in mouse auditory neurones. The Journal of physiology. 509 ( Pt 1):183-194.

22. Chen HM, DeLong CJ, Bame M, Rajapakse I, Herron TJ, Mclnnis MG, O'Shea KS (2014): Transcripts involved in calcium signaling and telencephalic neuronal fate are altered in induced pluripotent stem cells from bipolar disorder patients. Transl Psychiatry. 4:e375.

23. Ferreira MA, O'Donovan MC, Meng YA, Jones IR, Ruderfer DM, Jones L, Fan J, Kirov G, Perlis RH, Green EK, Smoller JW, Grozeva D, Stone J, Nikolov I, Chambert K, Hamshere ML, Nimgaonkar VL, Moskvina V, Thase ME, Caesar S, Sachs GS, Franklin J, Gordon-Smith K, Ardlie KG, Gabriel SB, Fraser C, Blumenstiel B, Defelice M, Breen G, Gill M, Morris DW, Elkin A, Muir WJ, McGhee KA, Williamson R, Maclntyre DJ, MacLean AW, St CD, Robinson M, Van Beck M, Pereira AC, Kandaswamy R, McQuillin A, Collier DA, Bass NJ, Young AH, Lawrence J, Ferrier IN, Anjorin A, Farmer A, Curtis D, Scolnick EM, McGuffin P, Daly MJ, Corvin AP, Holmans PA, Blackwood DH, Gurling HM, Owen MJ, Purcell SM, Sklar P, Craddock N, Wellcome Trust Case Control C (2008): Collaborative genome-wide association analysis supports a role for ANK3 and CACNA1C in bipolar disorder. Nature genetics. 40:1056-1058.

24. Lazarewicz MT, Migliore M, Ascoli GA (2002): A new bursting model of CA3 pyramidal cell physiology suggests multiple locations for spike initiation. Biosystems. 67:129-137.

25. Wheeler DW, Kullmann PH, Horn JP (2004): Estimating use-dependent synaptic gain in autonomic ganglia by computational simulation and dynamic-clamp analysis. Journal of neurophysiology. 92:26592671.

26. Morse TM, Carnevale NT, Mutalik PG, Migliore M, Shepherd GM (2010): Abnormal Excitability of Oblique Dendrites Implicated in Early Alzheimer's: A Computational Study. Front Neural Circuits. 4.

27. Goldman MS, Golowasch J, Marder E, Abbott LF (2001): Global structure, robustness, and modulation of neuronal models. The Journal of neuroscience : the official journal of the Society for Neuroscience. 21:5229-5238. 